\title{
The onset time of amiodarone-induced thyrotoxicosis (AIT) depends on AIT type
}

\author{
Luca Tomisti, Giuseppe Rossi², Luigi Bartalena', Enio Martino and Fausto Bogazzi \\ Endocrinology Unit, Department of Clinical and Experimental Medicine, University of Pisa, Ospedale Cisanello, \\ Via Paradisa, 2, 56124 Pisa, Italy, 'Endocrine Unit, Department of Clinical and Experimental Medicine, \\ University of Insubria, 21100 Varese, Italy and ${ }^{2}$ Unit of Epidemiology and Biostatistics, Institute of Clinical \\ Physiology, National Research Council, 56184 Pisa, Italy
}

Correspondence should be addressed to F Bogazzi Email fausto.bogazzi@med.unipi.it or fbogazzi@hotmail.com

\begin{abstract}
Objective: Considering the different pathogenic mechanisms of the two main forms of amiodarone-induced thyrotoxicosis (AIT), we ascertained whether this results in a different onset time as well.

Design and methods: We retrospectively analyzed the clinical records of 200 consecutive AIT patients (157 men and 43 women; mean age 62.2 \pm 12.6 years) referred to our Department from 1987 to 2012. The onset time of AIT was defined as the time elapsed from the beginning of amiodarone therapy and the first diagnosis of thyrotoxicosis, expressed in months. Factors associated with the onset time of AIT were evaluated by univariate and multivariate analyses.

Results: The median onset time of thyrotoxicosis was 3.5 months ( $95 \% \mathrm{Cl} 2-6$ months) in patients with type 1 AIT (AIT1) and 30 months (95\% Cl 27-32 months, $P<0.001$ ) in those with type 2 AIT (AIT2). Of the total number of patients, $5 \%$ with AIT1 and $23 \%$ with AIT2 $(P=0.007)$ developed thyrotoxicosis after amiodarone withdrawal. Factors affecting the onset time of thyrotoxicosis were the type of AIT and thyroid volume (TV).

Conclusions: The different pathogenic mechanisms of the two forms of AIT account for different onset times of thyrotoxicosis in the two groups. Patients with preexisting thyroid abnormalities (candidate to develop AIT1) may require a stricter follow-up during amiodarone therapy than those usually recommended. In AIT1, the onset of thyrotoxicosis after amiodarone withdrawal is rare, while AIT2 patients may require periodic tests for thyroid function longer after withdrawing amiodarone.
\end{abstract}

\section{Introduction}

Amiodarone-induced thyrotoxicosis (AIT) develops in $\sim 15 \%$ of patients under amiodarone therapy $(1,2)$. Two main forms of AIT may occur: type 1 is a form of iodineinduced hyperthyroidism occurring in patients with underlying thyroid abnormalities, and type 2 is a destructive thyroiditis mainly due to direct cytotoxic effects of amiodarone on thyroid follicular cells of a normal thyroid gland $(1,3,4,5)$. The prevalence of the two main forms of AIT has changed over the last 30 years in Italy with a current predominance of the type 2 AIT (AIT2) (6).

Occurrence of AIT is usually considered to be unpredictable, often sudden, and explosive, occurring either early or long after initiation of amiodarone treatment. In a group of 58 patients, Trip etal. (7) observed that average duration of amiodarone treatment before AIT occurrence was $\sim 3$ years, with a probability of $2.5 \%$ after 18 months and $33.5 \%$ after 48 months. In addition, Ahmed et al. (8) have recently reported an AIT incidence rate per 100 persons/year of 1.9 in a series of 303 patients taking amiodarone. Other small prospective studies described the onset of AIT after 12-47 months of amiodarone therapy $(9,10)$. AIT may also develop months after drug withdrawal, because of tissue storage of the drug and its metabolites and their slow release into the circulation.

Owing to the different pathogenic mechanisms of the two forms of AIT, we aimed at evaluating whether AIT1 and AIT2 may have different onset times in a large series of patients. This information might be useful in clinical 
practice for planning a different surveillance program of thyroid function.

\section{Materials and methods}

\section{Study design}

We retrospectively analyzed the clinical records of AIT patients referred to the Department of Clinical and Experimental Medicine, Endocrinology Section, University of Pisa, from January 1987 to December 2012.

Each patient gave her/his written informed consent, at the first clinical visit at our Department, for the use of anonymous data for research purpose. The Internal Review Board of our Department approved the study.

\section{Subjects and diagnosis of AIT}

A total of 200 consecutive AIT patients (157 men and 43 women; mean ( \pm s.D.), age $62.2 \pm 12.6$ years, range 24-87 years) were included in the study.

Diagnosis of AIT was based on clinical grounds (signs and symptoms of thyrotoxicosis) and laboratory findings, including increased serum free thyroxine $\left(\mathrm{FT}_{4}\right)$ and free tri-iodothyronine $\left(\mathrm{FT}_{3}\right)$ concentrations, undetectable serum TSH levels, and increased urinary iodine excretion (UIE). Diagnosis of AIT2 was based on the following criteria (1): normal or slightly increased TV without nodules $(\geq 1 \mathrm{~cm})$ at conventional ultrasonography, absent hypervascularity at color-flow Doppler sonography, absence of circulating thyroid-directed autoantibody (anti-thyroglobulin (TgAb), anti-thyroid peroxidase (TPOAb), anti-TSH receptor (TRAb)), and low/undetectable thyroid radioiodine uptake (RAIU) values $(<5 \%$ at $24 \mathrm{~h})$, as reported previously $(11,12)$.

The remaining subjects, including patients with Graves' disease, toxic adenoma, and multinodular goiter, did not meet the above criteria and were classified as type 1 AIT (AIT1). For the purpose of this study, all non-AIT2 patients were designated as AIT1, as reported previously (6). These criteria were applied retrospectively to patients diagnosed with AIT before 1990, when differentiation of the two main types of AIT had not yet been clearly established (6).

Clinical and biochemical findings of the two groups are given in Table 1.

\section{Time definitions}

Onset time of AIT was defined as the period elapsed from initiation of amiodarone therapy and first diagnosis of
Table 1 Clinical and biochemical features of the study groups. Data are expressed as mean \pm s.D. Normal values in our laboratory are as follows: $\mathrm{FT}_{4}, 7-17 \mathrm{pg} / \mathrm{ml} \mathrm{FT}_{3}, 2.7-4.5 \mathrm{pg} / \mathrm{ml}$; TSH, 0.4-3.4 mU/l; TPOAb, < $10 \mathrm{lU} / \mathrm{ml}$; and 3rd and 24th h RAIU, in our area, $15-30$ and $30-45 \%$ respectively. To convert serum $\mathrm{FT}_{4}$ and $\mathrm{FT}_{3}$ values from $\mathrm{pg} / \mathrm{ml}$ to $\mathrm{pmol} / \mathrm{l}$, multiply by 1.29 and 1.54 respectively.

\begin{tabular}{|c|c|c|c|}
\hline Variable & $\begin{array}{l}\text { AIT1 }(n=42) \\
\text { mean } \pm \text { s.D. }\end{array}$ & $\begin{array}{c}\text { AIT2 }(n=158) \\
\text { mean } \pm \text { s.D. }\end{array}$ & $\boldsymbol{P}$ \\
\hline Sex (female/male) & $11 / 31$ & $32 / 126$ & 0.41 \\
\hline Age (years) & $65.3 \pm 10.6$ & $61.4 \pm 13$ & 0.07 \\
\hline BSA $\left(m^{2}\right)$ & $1.85 \pm 0.23$ & $1.88 \pm 0.21$ & 0.52 \\
\hline $\mathrm{BMI}$ & $26.1 \pm 4.6$ & $25.4 \pm 3.9$ & 0.35 \\
\hline Basal $\mathrm{FT}_{4}(\mathrm{pg} / \mathrm{ml})$ & $28.2 \pm 15.3$ & $41.7 \pm 16.4$ & $<0.001$ \\
\hline Basal $\mathrm{FT}_{3}(\mathrm{pg} / \mathrm{ml})$ & $7.2 \pm 4.7$ & $10.2 \pm 5.2$ & $<0.001$ \\
\hline $\mathrm{FT}_{4} / \mathrm{FT}_{3}$ & $4.2 \pm 2.2$ & $4.4 \pm 1.3$ & 0.50 \\
\hline TSH (mIU/l) & $<\overline{0.01}$ & $<\overline{0.01}$ & 1.00 \\
\hline TPOAb (IU/ml) & $138 \pm 384$ & $<10$ & $<0.001$ \\
\hline UIE $(\mu g / I)$ & $5769 \pm 10999$ & $7967 \pm 8045$ & 0.24 \\
\hline TG (ng/ml) & $218.3 \pm 492.2$ & $136.1 \pm 203.9$ & 0.32 \\
\hline 3rd h RAIU (\%) & $7.1 \pm 8$ & $1.5 \pm 1.1$ & $<0.001$ \\
\hline 24th h RAIU (\%) & $12.8 \pm 13.3$ & $1.1 \pm 1.3$ & $<0.001$ \\
\hline $\mathrm{TV}(\mathrm{ml})$ & $56.7 \pm 39.7$ & $19.6 \pm 8.8$ & $<0.001$ \\
\hline TV norm $\left(\mathrm{ml} / \mathrm{m}^{2}\right)$ & $30.5 \pm 20.1$ & $10.5 \pm 4.6$ & $<0.001$ \\
\hline $\begin{array}{l}\text { CFDS pattern } \\
(0 / 1 / 2 / 3)\end{array}$ & $3 / 1 \overline{1 / 23 / 5}$ & $154 / 4 / 0 / 0$ & $<0.001$ \\
\hline $\begin{array}{l}\text { Duration of } \\
\text { amiodarone } \\
\text { treatment } \\
\text { (months) }\end{array}$ & $9.9 \pm 11.8$ & $28.7 \pm 16.1$ & $<0.001$ \\
\hline $\begin{array}{l}\text { Daily dose of amio- } \\
\text { darone (mg) }\end{array}$ & $193 \pm 38$ & $192 \pm 54$ & 0.96 \\
\hline $\begin{array}{c}\text { Cumulative dose of } \\
\text { amiodarone (g) }\end{array}$ & $46.8 \pm 31.7$ & $134.4 \pm 108.8$ & 0.001 \\
\hline AIT post AMIO (\%) & $2 / 40(4.76)$ & $36 / 122$ (22.8) & 0.007 \\
\hline
\end{tabular}

Basal $\mathrm{FT}_{4}$ and basal $\mathrm{FT}_{3}$, serum thyroid hormone concentrations at diagnosis; UIE, urinary iodine excretion; TG, serum thyroglobulin; 3rd and 24th h RAIU, 3rd and 24th h thyroid radioiodine uptake; BSA, body surface area calculated using the Mosteller formula, as described previously; TV, thyroid volume estimated by ultrasonography; TV norm, normalized thyroid volume obtained by dividing thyroid volume by body surface area; CFDS pattern, color-flow Doppler sonography pattern expressed as the number of patients having pattern 0/1/2/3; AIT after AMIO, number of patients (and percentage) developing AIT after amiodarone withdrawal.

thyrotoxicosis. Onset time after amiodarone withdrawal was defined as the period elapsed from the withdrawal of amiodarone therapy and first diagnosis of thyrotoxicosis in patients who developed thyrotoxicosis after amiodarone discontinuation.

\section{Thyroid status}

Serum $\mathrm{FT}_{4}, \mathrm{FT}_{3}$ (Vitros Immunodiagnostics, The Broadway, Amersham), TSH (Immulite 2000, third generation TSH; Diagnostic Products Corp., Los Angeles, CA, USA), 
TG (Access Immunoassay Systems; Beckman Coulter, Inc., Brea, CA, USA), TRAb (TRAK human; Brahms, Hennigsdorf, Germany), TgAb (AIA-Pack TgAb; Tosoh, Tokyo, Japan), and TPOAb (AIA-Pack TPOAb; Tosoh) were assayed using commercial kits. Normal values in our laboratories are as follows: $\mathrm{FT}_{4}, 7-17 \mathrm{pg} / \mathrm{ml}(9.0-22.0 \mathrm{pmol} / \mathrm{l}) ; \mathrm{FT}_{3}$, 2.7-4.5 pg/ml (4.2-7.0 pmol/1); TSH, 0.4-3.4 mU/1; TRAb, $<1 \mathrm{U} / \mathrm{l}$; TgAb, <30 IU/ml; and TPOAb, <10 IU/ml.

Random morning urinary samples were collected for iodine measurements using an autoanalyzer apparatus (Technicon, Rome, Italy). Median UIE in our area is $110 \mu \mathrm{g} / \mathrm{l}$.

\section{Thyroid ultrasound and RAIU}

TV was measured by ultrasonography and calculated by the ellipsoid model (width $\times$ length $\times$ thickness $\times 0.52$ for each lobe) as described previously $(13,14)$. TV was normalized by body surface area (TV norm) calculated using the Mosteller

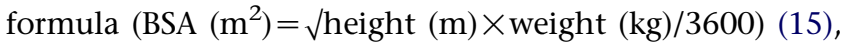
because, as reported previously (16), BSA accounts for the main variations in TVs, including sex-related differences; normal values in our areas are $3.5-13 \mathrm{ml} / \mathrm{m}^{2}$.

Thyroid RAIU was measured at 3 and $24 \mathrm{~h}$ after the administration of a tracer dose $(50 \mu \mathrm{Ci})$ of ${ }^{131} \mathrm{I}$. The normal 3 and $24 \mathrm{~h}$ RAIU values in our area are $10-20$ and $30-45 \%$ respectively.

\section{Statistical analysis}

Results are expressed as mean \pm s.D. for quantitative data and percentage for categorical data. The comparison between the two study groups (AIT1 vs AIT2) for clinical and biochemical features was performed by the Wilcoxon rank-sum test for quantitative variables and by Fisher's exact test for categorical variables. The onset time of AIT was analyzed by the Kaplan-Mayer survival curve and the comparison between groups was performed by the log-rank test. Factors associated with onset time were evaluated by univariate and multivariate analyses using the Cox regression model. Hazard ratio (HR) and 95\% CI were also reported. A two-sided $P$ value of $<0.05$ was considered statistically significant. Statistical analysis was performed using the JMP 4 (SAS Institute, Inc., Cary, NC, USA) software.

\section{Results}

Out of 200 patients evaluated in this study, 42 (21\%) were diagnosed with AIT1 and 158 (79\%) with AIT2. The clinical and biochemical features of the two groups of patients are given in Table 1 . As expected, patients with AIT2 had lower 3 and 24-h RAIU values and a smaller TV than those with AIT1. In addition, patients with AIT2 had significantly higher serum thyroid hormone concentrations $\left(\mathrm{FT}_{4} 41.7 \pm 16.4 \mathrm{pg} / \mathrm{ml}\right.$ and $\left.\mathrm{FT}_{3} 10.2 \pm 5.2 \mathrm{pg} / \mathrm{ml}\right)$ than those with AIT1 $\left(\mathrm{FT}_{4} 28.2 \pm 15.3 \mathrm{pg} / \mathrm{ml}\right.$ and $\mathrm{FT}_{3} 7.2 \pm$ $4.7 \mathrm{pg} / \mathrm{ml}, P<0.001$ and $P<0.001$ respectively). Eight patients with AIT1 had Graves' disease, six a toxic adenoma, and 28 a multinodular goiter.

Median onset time of thyrotoxicosis in the AIT1 group was 3.5 months (range 1-61 months) and 30 months in AIT2 group (range 1-95 months, log-rank <0.001, Fig. 1). Distribution of onset time of AIT in the two groups of patients is shown in Fig. 2.

Out of 200 patients, 38 (19\%) developed thyrotoxicosis after amiodarone withdrawal: two patients in the AIT1 group (4.8\%) and 36 patients in the AIT2 group (22.9\%, $P=0.007)$. The two patients with AIT1 developed thyrotoxicosis at 1 and 12 months (median time 6.5 months) after the withdrawal of amiodarone. The time elapsed from the withdrawal of amiodarone therapy and the first diagnosis of thyrotoxicosis in the AIT2 group (median time 5.5 months, range 1-18 months) is summarized in Fig. 3. In the AIT2 group, no difference was found between patients developing thyrotoxicosis before or after amiodarone withdrawal in TV, normalized TV, BMI, and sex, whereas patients developing thyrotoxicosis after amiodarone withdrawal were significantly younger $(56.8 \pm 11.9$ vs $62.8 \pm 13$ years respectively; $P=0.014)$. In addition, the onset time of thyrotoxicosis

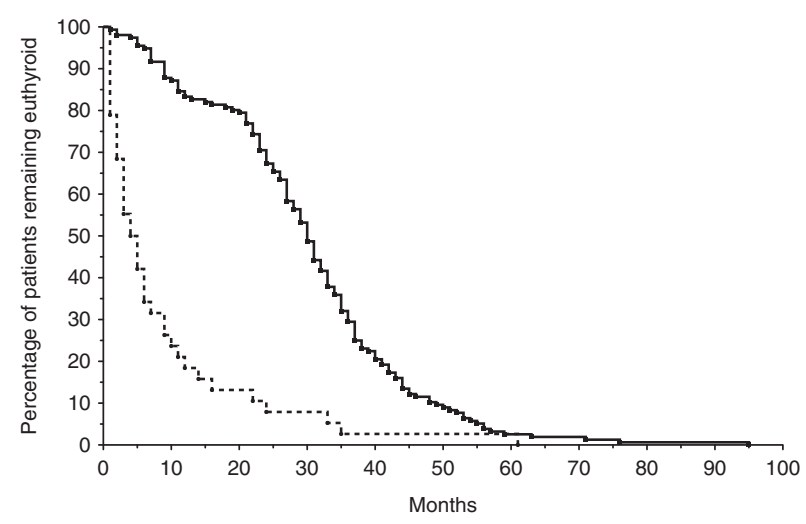

\section{Figure 1}

The Kaplan-Meier event-free survival estimates the onset time of thyrotoxicosis in type 1 AIT (dotted line) and type 2 AIT (continuous line). Onset time of thyrotoxicosis was significantly shorter in type 1 AIT patients (log rank, $P<0.001$ ). 

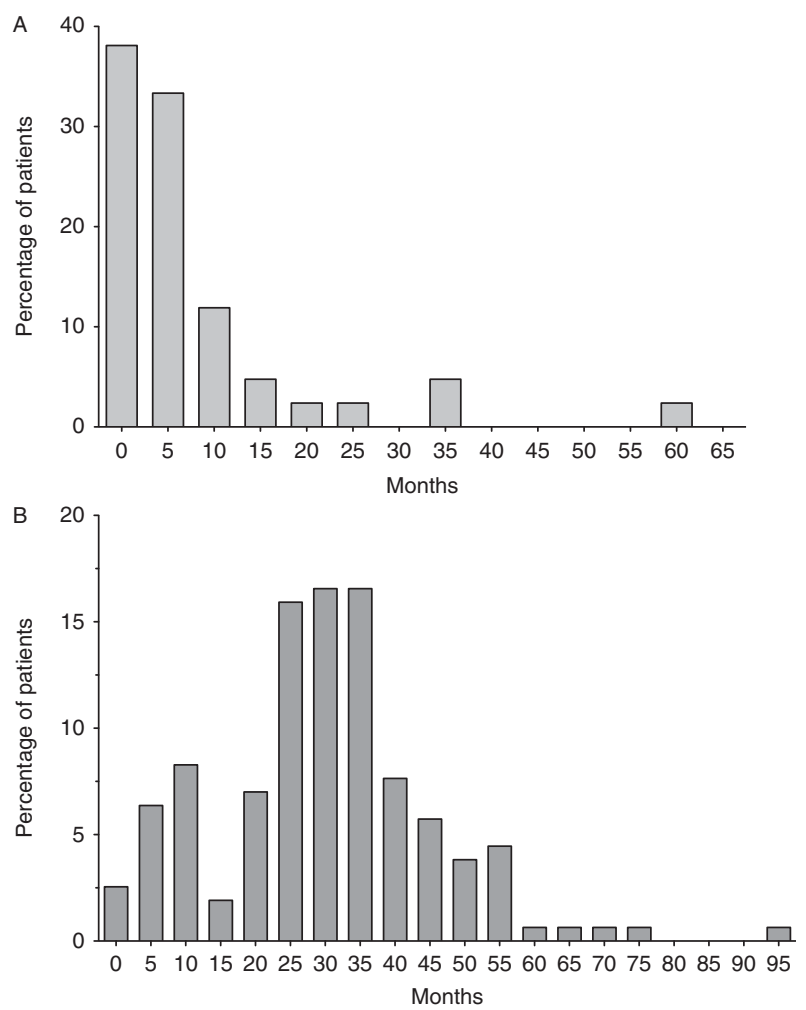

\section{Figure 2}

Percentage of patients developing amiodarone-induced thyrotoxicosis (AIT) from the beginning of amiodarone therapy in type 1 AIT ( $n=42$ patients, A) and type 2 AIT ( $n=158$ patients, B).

did not significantly differ in patients who developed AIT during amiodarone therapy or after therapy withdrawal (median time 31 months, range 1-200 months, and median time 26 months, range 2-63 months respectively; $\log$-rank $P=0.066$ ).

Factors affecting the onset time of thyrotoxicosis were evaluated by the univariate and multivariate analyses, as reported in Table 2 . In the univariate analysis, a shorter onset time was related to AIT1 $(P<0.0001)$ and to a larger normalized TV $(P<0.0001)$. When patients were divided based on the type of AIT, the univariate analysis confirmed the significant effect of the normalized TV on the onset time of thyrotoxicosis (HR 1.01, 95\% CI 1.00-1.03, $P=0.04$ in the AIT1 group and HR 1.05, 95\% CI 1.01$1.09, P=0.01$ in the AIT2 group).

After adjusting for age, sex, and BMI, in the multivariate analysis, the type of AIT and the normalized TV were confirmed as the main independent factors affecting the onset time of thyrotoxicosis with a HR of 2.88 (95\% CI
$1.76-4.55, P<0.0001)$ and $1.03(95 \%$ CI $1.01-1.04$, $P<0.0001)$ per unit of increment respectively. No interaction was found between the type of AIT and normalized TV $(P=0.42)$.

\section{Discussion}

Onset of thyrotoxicosis during amiodarone therapy is usually considered to be unpredictable $(1,3,7)$ and has been reported to occur at any time during therapy as well as after drug withdrawal. However, no studies have so far investigated as to whether differences exist in the onset time of the two main forms of AIT.

The present retrospective study of a large cohort of patients is the first report of a significant difference in the onset time of thyrotoxicosis of the two forms of AIT. The time elapsed from initiation of amiodarone therapy and occurrence of thyrotoxicosis was much shorter in AIT1 than in AIT2, the median time being 3.5 and 30 months respectively.

This observation is consistent with previous limited observations in small series and the different pathogenic mechanisms of the two forms of AIT $(6,12,17)$. AIT1 is a form of iodine-induced hyperthyroidism arising in a thyroid gland with underlying functional autonomy; in these patients, iodine load may rapidly trigger an increased thyroid hormone synthesis. Conversely, AIT2, being a destructive thyroiditis due to a direct cytotoxic effect of amiodarone or iodine $(1,3,18,19)$, may imply that a high intrathyroid drug concentration may be reached before the damage of thyroid follicular cells becomes evident at a clinical level (20).

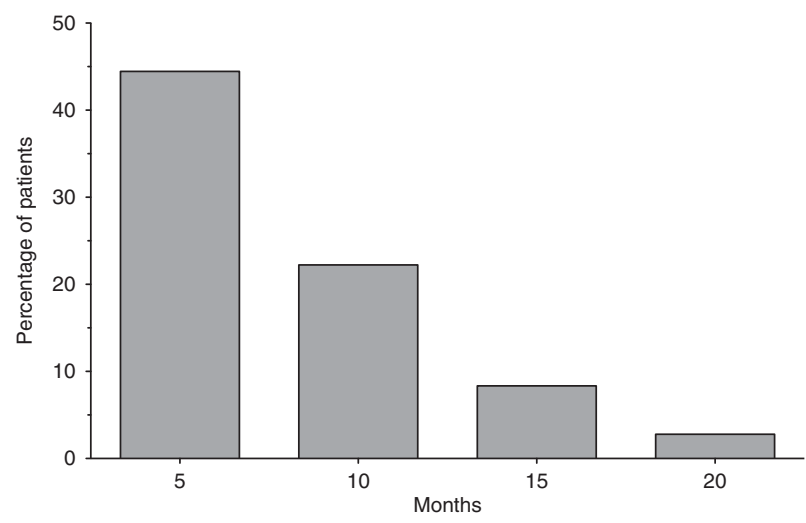

Figure 3

Time distance from the withdrawal of amiodarone therapy and the onset of thyrotoxicosis in the 36 type 2 AIT patients developing thyrotoxicosis after amiodarone withdrawal. 
Table 2 Factors associated with the onset time, evaluated by the Cox regression model. Factors associated with the onset time were evaluated using univariate analysis by the Cox regression model.

\begin{tabular}{|c|c|c|c|}
\hline \multirow[b]{2}{*}{ Variables } & \multicolumn{3}{|c|}{ Univariate } \\
\hline & $\mathrm{HR}$ & $95 \% \mathrm{Cl}$ & $P$ \\
\hline AIT type & 4.20 & $2.90-5.96$ & $<0.0001$ \\
\hline Age (years) & 0.99 & $0.98-1.00$ & 0.435 \\
\hline Sex & 0.81 & $0.58-1.16$ & 0.257 \\
\hline BMI & 1.02 & $0.98-1.05$ & 0.206 \\
\hline TV norm & 1.05 & $1.03-1.06$ & $<0.0001$ \\
\hline
\end{tabular}

Type 1 AIT, a larger thyroid volume, and a larger body surface area were associated with a shorter onset time; BSA, body surface area calculated using the Mosteller formula, as described previously; TV norm, normalized thyroid volume obtained by dividing thyroid volume by body surface area; $\mathrm{HR}$, hazard ratio.

However, AIT2 may also have an abrupt and explosive onset at any time during amiodarone therapy (21). In fact, although the mean onset time was 30 months in AIT2 patients, $\sim 15 \%$ of subjects developed thyrotoxicosis after $<12$ months of amiodarone therapy.

In addition, we observed that $\sim 20 \%$ of AIT2 patients developed thyrotoxicosis after amiodarone withdrawal, probably due to the long storage time and the slow release of amiodarone and its main metabolite, desethylamiodarone, from the adipose tissue, which prolong and maintain exposure of the thyroid gland to amiodarone. This is in keeping with the observation that onset time of thyrotoxicosis in this subset of patients did not differ significantly from that of patients who developed thyrotoxicosis during amiodarone therapy. This event was rarely observed in AIT1 $(<5 \%)$, which is associated with an earlier occurrence of thyrotoxicosis.

Despite the clear-cut difference in the average onset time of AIT1 and AIT2, there was a partial overlap between the two forms. A possible reason for the presence of a longer onset time in a small subset of AIT1 patients may be a concomitant destructive process (mixed forms) accountable for a longer onset time. However, 70\% of AIT1 patients but only $5 \%$ of AIT2 patients developed AIT within 6 months. At variance, in $70 \%$ of AIT2 patients, thyrotoxicosis developed after 2 years, while only $10 \%$ of AIT1 has such a long onset time. The interval between initiation of amiodarone therapy and occurrence of AIT, nevertheless, cannot be used, alone, to distinguish the two forms of AIT in individual patients.

Another independent determinant of the onset time of thyrotoxicosis was the TV. We have previously reported that the normalized TV is an independent factor affecting the response to glucocorticoid therapy in AIT2 patients (11). In this study, we have found that a larger TV was related to a shorter onset time of thyrotoxicosis, confirming that the thyroid size plays a role in development and severity of AIT. It is noteworthy that the TV is calculated at the moment of the diagnosis of AIT. This is a limitation of our study due to its retrospective design. However, no data regarding modification of the TV in patients under amiodarone therapy have been currently reported. Thus, being so we have considered the TV, estimated at the time of diagnosis of AIT, to be a good proxy for the basal TV. As a matter of fact, a prospective study by Silva et al. (22) did not find any correlation among the time of use, the daily dose and the cumulative dose of amiodarone, and the detection of goiter in a subset of patients taking amiodarone because of Chagas disease. This information may support the concept that TV should not be significantly affected by the amiodarone therapy.

It has been previously observed that a lower BMI may be an independent risk factor for the development of AIT (23). However, our study failed to find a relationship between the onset time of thyrotoxicosis and BMI. It is worth noting that patients described in the study by Stan et al. (23) had congenital cardiomyopathy and a normal to low BMI, at variance with patients of the present series.

In conclusion, our data showed that patients with AIT1 have a shorter median onset time of thyrotoxicosis than those with AIT2. In patients under amiodarone therapy, evaluation of thyroid function is usually recommended at 6-month intervals $(1,2,3,24)$. Based on the present data, we suggest a closer follow-up (i.e. every 1-3 months) for the patients with preexisting thyroid abnormalities (autonomous multinodular goiter or latent Graves' disease) who may develop AIT1.

By contrast, in patients with a normal thyroid gland we cannot provide a follow-up plan able to guarantee an early diagnosis of AIT because of the large variability of their onset time. In addition, the onset of thyrotoxicosis is often sudden and the clinical features of thyrotoxicosis may be atypical and mild, mainly in older patients. As a result, evaluation of thyroid function should be performed, at any time, in patients under amiodarone therapy showing an unexpected worsening of cardiac conditions and in patients, also taking warfarin therapy, showing an unexplained increased sensitivity to anticoagulant therapy (25).

However, in patients under amiodarone therapy showing no signs of thyrotoxicosis, we believe that a thyroid function test should be periodically performed because the diagnosis of thyrotoxicosis might be strongly delayed by the atypical clinical features often observed. 
Finally, thyroid function should be monitored for at least 2 years after amiodarone withdrawal, particularly in patients without apparent thyroid abnormalities.

\section{Declaration of interest}

The authors declare that there is no conflict of interest that could be perceived as prejudicing the impartiality of the research reported.

\section{Funding}

This research did not receive any specific grant from any funding agency in the public, commercial or not-for-profit sector.

\section{References}

1 Martino E, Bartalena L, Bogazzi F \& Braverman LE. The effects of amiodarone on the thyroid. Endocrine Reviews 200122 240-254. (doi:10.1210/edrv.22.2.0427)

2 Bogazzi F, Bartalena L, Gasperi M, Braverman LE \& Martino E. The various effects of amiodarone on thyroid function. Thyroid $2001 \mathbf{1 1}$ 511-519. (doi:10.1089/105072501300176471)

3 Eskes SA \& Wiersinga WM. Amiodarone and thyroid. Best Practice \& Research. Clinical Endocrinology \& Metabolism 200923 735-751. (doi:10.1016/j.beem.2009.07.001)

4 Bogazzi F, Tomisti L, Bartalena L, Aghini-Lombardi F \& Martino E. Amiodarone and the thyroid: a 2012 update. Journal of Endocrinological Investigation 201235 340-348. (doi:10.3275/8298)

5 Barbesino G. Drugs affecting thyroid function. Thyroid 201020 763-770. (doi:10.1089/thy.2010.1635)

6 Bogazzi F, Bartalena L, Dell'Unto E, Tomisti L, Rossi G, Pepe P, Tanda ML, Grasso L, Macchia E, Aghini-Lombardi F et al. Proportion of type 1 and type 2 amiodarone-induced thyrotoxicosis has changed over a 27-year period in Italy. Clinical Endocrinology 200767 533-537. (doi:10.1111/j.1365-2265.2007.02920.x)

7 Trip MD, Wiersinga W \& Plomp TA. Incidence, predictability, and pathogenesis of amiodarone-induced thyrotoxicosis and hypothyroidism. American Journal of Medicine 199191 507-511. (doi:10.1016/ 0002-9343(91)90187-3)

8 Ahmed S, Van Gelder IC, Wiesfeld AC, Van Veldhuisen DJ \& Links TP. Determinants and outcome of amiodarone-associated thyroid dysfunction. Clinical Endocrinology 201175 388-394. (doi:10.1111/ j.1365-2265.2011.04087.x)

9 Martino E, Aghini-Lombardi F, Bartalena L, Grasso L, Loviselli A, Velluzzi F, Pinchera A \& Braverman LE. Enhanced susceptibility to amiodarone-induced hypothyroidism in patients with thyroid autoimmune disease. Archives of Internal Medicine 1994154 2722-2726. (doi:10.1001/archinte.1994.00420230115013)

10 Mariotti S, Loviselli A, Murenu S, Sau F, Valentino L, Mandas A Vacquer S, Martino E, Balestrieri A \& Lai ME. High prevalence of thyroid dysfunction in adult patients with $\beta$-thalassemia major submitted to amiodarone treatment. Journal of Endocrinological Investigation 199922 55-63. (doi:10.1007/BF03345479)
11 Bogazzi F, Bartalena L, Tomisti L, Rossi G, Tanda ML, Dell'Unto E, Aghini-Lombardi F \& Martino E. Glucocorticoid response in amiodarone-induced thyrotoxicosis resulting from destructive thyroiditis is predicted by thyroid volume and serum free thyroid hormone concentrations. Journal of Clinical Endocrinology and Metabolism 200792 556-562. (doi:10.1210/jc.2006-2059)

12 Eaton SE, Euinton HA, Newman CM, Weetman AP \& Bennet WM. Clinical experience of amiodarone-induced thyrotoxicosis over a 3-year period: role of colour-flow Doppler sonography. Clinical Endocrinology 200256 33-38. (doi:10.1046/j.0300-0664.2001.01457.x)

13 Brunn J, Block U, Ruf G, Bos I, Kunze WP \& Scriba PC. Volumetric analysis of thyroid lobes by real-time ultrasound. Deutsche Medizinische Wochenschrift 1981106 1338-1340. (doi:10.1055/s-2008-1070506)

14 Knudsen N, Bols B, Bulow I, Jorgensen T, Perrild H, Ovesen L \& Laurberg P. Validation of ultrasonography of the thyroid gland for epidemiological purposes. Thyroid 19999 1069-1074. (doi:10.1089/ thy.1999.9.1069)

15 Mosteller RD. Simplified calculation of body-surface area. New England Journal of Medicine 1987317 1098. (doi:10.1056/NEJM1987102231 71717)

16 Gomez JM, Maravall FJ, Gomez N, Guma A \& Soler J. Determinants of thyroid volume as measured by ultrasonography in healthy adults randomly selected. Clinical Endocrinology 200053 629-634. (doi:10.1111/j.1365-2265.2000.01138.x)

17 Cohen-Lehman J, Dahl P, Danzi S \& Klein I. Effects of amiodarone therapy on thyroid function. Nature Reviews. Endocrinology 20106 34-41. (doi:10.1038/nrendo.2009.225)

18 Pearce EN, Farwell AP \& Braverman LE. Thyroiditis. New England Journal of Medicine 2003348 2646-2655. (doi:10.1056/NEJMra021194)

19 Bartalena L, Brogioni S, Grasso L, Bogazzi F, Burelli A \& Martino E. Treatment of amiodarone-induced thyrotoxicosis, a difficult challenge: results of a prospective study. Journal of Clinical Endocrinology and Metabolism 199681 2930-2933. (doi:10.1210/jcem.81.8.8768854)

20 Bogazzi F, Raggi F, Ultimieri F, Russo D, Cosci C, Cecchetti P, Dell'Unto E, Sardella C, Tonacchera M, Vitti P et al. Uptake of amiodarone by thyroidal and non-thyroidal cell lines. Journal of Endocrinological Investigation 200629 61-66. (doi:10.1007/BF03349178)

21 Tsang W \& Houlden RL. Amiodarone-induced thyrotoxicosis: a review. Canadian Journal of Cardiology 200925 421-424. (doi:10.1016/S0828282X(09)70512-4)

22 Silva JR, Guariento ME, Fernandes GA, Maciel RM \& Ward LS. Impact of long-term administration of amiodarone on the thyroid function of patients with Chagas' disease. Thyroid 200414 371-377. (doi:10.1089/ 105072504774193212)

23 Stan MN, Ammash NM, Warnes CA, Brennan MD, Thapa P, Nannenga MR \& Bahn RS. Body mass index and the development of amiodarone-induced thyrotoxicosis in adults with congenital heart disease - a cohort study. International Journal of Cardiology 2012167 821-826. (doi:10.1016/j.ijcard.2012.02.015)

24 Raebel MA, Carroll NM, Simon SR, Andrade SE, Feldstein AC, Lafata JE Nelson WW, Chan KA, Gunter MJ, Tolsma D et al. Liver and thyroid monitoring in ambulatory patients prescribed amiodarone in 10 HMOs. Journal of Managed Care Pharmacy 200612 656-664.

25 Tomisti L, Del Re M, Bartalena L, Tanda ML, Pucci A, Pambianco F, Danesi R, Braverman LE, Martino E \& Bogazzi F. Effects of amiodarone, thyroid hormones and CYP2C9 and VKORC1 polymorphisms on warfarin metabolism: a review of the literature. Endocrine Practice 201319 1043-1049. (doi:10.4158/EP13093.RA)

Received 2 April 2014

Revised version received 30 May 2014

Accepted 16 June 2014 